\title{
Localized intersection of currents and the Lefschetz coincidence point theorem
}

\author{
Cinzia Bisi · Filippo Bracci · Takeshi Izawa · Tatsuo Suwa
}

Received: 3 June 2014 / Accepted: 21 January 2015 / Published online: 6 February 2015

(C) Fondazione Annali di Matematica Pura ed Applicata and Springer-Verlag Berlin Heidelberg 2015

\begin{abstract}
We introduce the notion of a Thom class of a current and define the localized intersection of currents. In particular, we consider the situation where we have a $C^{\infty}$ map of manifolds and study localized intersections of the source manifold and currents on the target manifold. We then obtain a residue theorem on the source manifold and give explicit formulas for the residues in some cases. These are applied to the problem of coincidence points of two maps. We define the global and local coincidence homology classes and indices. A representation of the Thom class of the graph as a Čech-de Rham cocycle immediately gives us an explicit expression of the index at an isolated coincidence point, which in turn gives explicit coincidence classes in some non-isolated components. Combining these, we have a general coincidence point theorem including the one by S. Lefschetz.
\end{abstract}

Cinzia Bisi was partially supported by PRIN2010-2011 Protocollo: 2010NNBZ78-012, by Firb2012 Codice: RBFR12W1AQ-001 and by GNSAGA-INDAM. Filippo Bracci was partially supported by the ERC Grant "HEVO - Holomorphic Evolution Equations" n. 277691. Tatsuo Suwa was partially supported by the JSPS Grant No. 24540060.

C. Bisi

Dipartimento di Matematica ed Informatica, Università di Ferrara,

Via Machiavelli, n. 35, 44121 Ferrara, Italy

e-mail: bsicnz@unife.it

F. Bracci

Dipartimento di Matematica, Università di Roma "Tor Vergata", Via della Ricerca Scientifica, n. 1, 00133 Rome, Italy

e-mail: fbracci@mat.uniroma2.it

T. Izawa

Department of Information and Computer Science, Hokkaido University of Science,

Sapporo 006-8585, Japan

e-mail: t-izawa@hus.ac.jp

T. Suwa $(\bowtie)$

Department of Mathematics, Hokkaido University, Sapporo 060-0810, Japan

e-mail: tsuwa@sci.hokudai.ac.jp 
Keywords Alexander duality - Thom class - Localized intersections - Residue theorem · Coincidence classes and indices · Lefschetz Coincidence point formula

Mathematics Subject Classification $14 \mathrm{C} 17 \cdot 32 \mathrm{C} 30 \cdot 37 \mathrm{C} 25 \cdot 55 \mathrm{M} 05 \cdot 55 \mathrm{M} 20 \cdot 57 \mathrm{R} 20$. 58A25

\section{Introduction}

For two cycles in a manifold, the localized intersection product of their classes is defined, in the homology of their set theoretical intersection, via Alexander dualities and the cup product in the relative cohomology. Thus for a cycle $C$ in a manifold $W$, the corresponding class in the relative cohomology carries the local information on $C$. For a submanifold $M$ of $W$, this is the Thom class of $M$, which may be identified with the Thom class of the normal bundle of $M$ in $W$ by the tubular neighborhood theorem. In this paper, we take up the localization problem of currents. We introduce the notion of a Thom class of a current and define the localized intersection of two currents. In particular, we consider the intersections of a fixed submanifold $M$ and currents on $W$, obtain a residue theorem on $M$ and give explicit expressions of the residues in some cases (see Theorems 4.9 and 4.14 below for precise statements). As an application, we study the coincidence point problem for two maps.

The coincidence point formula discovered by Lefschetz (cf. [6,7]) is formulated for a pair of continuous maps between compact oriented topological manifolds of the same dimension. Using the above, we define global and local homology classes of coincidence for a pair of $C^{\infty}$ maps $M \rightarrow N$ and give a general coincidence point theorem, even in the case the dimensions $m$ and $n$ of $M$ and $N$ are different $(m \geq n)$ and the coincidence points are non-isolated (Definitions 5.1 and 5.2). In the case $m=n$, the use of Thom class in the Čech-de Rham cohomology immediately gives us an explicit expression of the coincidence index at an isolated coincidence point (Propositions 5.5). This gives in turn an explicit coincidence homology class at a certain non-isolated coincidence component in the case $m>n$ (Proposition 5.7). We then have a general coincidence point formula, including the Lefschetz coincidence point formula.

The paper is organized as follows. In Sect. 3, we recall preliminary materials such as Poincaré and Alexander dualities, global and localized intersection products, Thom classes in various settings and an explicit expression, in the Čech-de Rham cohomology, of the Thom class of an oriented vector bundle (Proposition 3.3). In Sect. 4, we consider the localization problem of currents, introduce the notion of a Thom class of a current and give some examples. We then consider localized intersections of currents. In particular, we study intersections of a fixed submanifold $M$ in a manifold $W$ and currents on $W$. In fact, we consider a more general situation where we have a map $F: M \rightarrow W$ (Definition 4.7). For a closed current $T$ on $W$, we have the intersection product $M \cdot_{F} T$ in the homology of $M$, and if $T$ is localized at a compact set $\tilde{S}$ in $W$ and if $\Psi_{T}$ is a Thom class of $T$ along $\tilde{S}$, we have the residue of $F^{*} \Psi_{T}$ in the homology of $S=F^{-1} \tilde{S}$ as a localized intersection product. If $S$ has several connected components, we have a "residue theorem" (Theorem 4.9). We give an explicit formula for the residue at a non-isolated component of $S$ in the case it is a submanifold of $M$ (Theorem 4.14). These are conveniently used in Sect. 5 , where we study the coincidence point problem for two maps.

Let $M$ and $N$ be manifolds of dimensions $m$ and $n$ and let $f, g: M \rightarrow N$ be two maps. We define the global coincidence class of the pair $(f, g)$ in the $(m-n)$-th homology of $M$ (Definition 5.1). If $M$ is compact, we also define the local coincidence class of the pair in the 
$(m-n)$-th homology of the set of points in $M$ where $f$ and $g$ coincide (Definition 5.2). We then apply Theorem 4.9 to get a general coincidence point theorem (Theorem 5.4). In the case $m=n$, we have a formula for the coincidence index at an isolated coincidence point as the local mapping degree of $g-f$ (Proposition 5.5). This is, in fact, a classical result; however, we give a short direct proof using the aforementioned expression of the Thom class in the Čech-de Rham cohomology. This together with Theorem 4.14 gives an explicit expression of the coincidence homology class at a non-isolated component (Proposition 5.7). If $M$ and $N$ are compact manifolds of the same dimension, we have a general coincidence point formula (Theorem 5.9), which reduces to the Lefschetz coincidence point formula in the case the coincidence points are isolated (Corollary 5.10).

\section{Notation and conventions}

For a topological space $X$, we denote by $H_{*}(X, \mathbb{C})$ and $H^{*}(X, \mathbb{C})$ its homology and cohomology of finite singular chains with $\mathbb{C}$ coefficients. Also we denote by $\breve{H}_{*}(X, \mathbb{C})$ the homology of locally finite singular chains (Borel-Moore homology). For a $C^{\infty}$ manifold, they can be computed using $C^{\infty}$ simplicial chains. To be a little more precise, any $C^{\infty}$ manifold $M$ admits a $C^{\infty}$ triangulation, which is essentially unique, and the groups $H_{*}(M, \mathbb{C})$ and $H^{*}(M, \mathbb{C})$ are naturally isomorphic with the ones defined by finite $C^{\infty}$ simplicial chains. Also the group $\breve{H}_{*}(M, \mathbb{C})$ is naturally isomorphic with the homology of locally finite $C^{\infty}$ simplicial chains.

In the sequel, a locally finite $C^{\infty}$ simplicial chain is simply called a chain, unless otherwise stated. Thus, a chain $C$ is expressed as a locally finite sum $C=\sum a_{i} s_{i}$ with $a_{i}$ in $\mathbb{C}$ and $s_{i}$ oriented $C^{\infty}$ simplices. We set $|C|=\bigcup s_{i}$ and call it the support of $C$. It is a closed set. For a cycle $C$, its class in the homology of the ambient space is denoted by $[C]$, while the class in the homology of its support is simply denoted by $C$.

For an open set $U$ in $M$, we denote by $A^{p}(U)$ and $A_{c}^{p}(U)$, respectively, the spaces of complex valued $C^{\infty} p$-forms and $p$-forms with compact support on $U$. The cohomology of the complex $\left(A^{*}(M), d\right)$ is the de Rham cohomology $H_{\mathrm{dR}}^{*}(M)$ and that of $\left(A_{c}^{*}(M), d\right)$ is the cohomology $H_{c}^{*}(M)$ with compact support. A $C^{\infty}$ form will be simply called a form unless otherwise stated.

\section{3 Čech-de Rham cohomology and the Thom class}

For the background on the Čech-de Rham cohomology, we refer to [2]. The integration theory on this cohomology is developed in [8]. See [12] also for these materials and for the description of the Thom class in the framework of relative Čech-de Rham cohomology. The relation with the combinatorial viewpoint, as given in [3], is discussed in [13].

In this section, we let $M$ denote a $C^{\infty}$ manifold of dimension $m$.

\subsection{Poincaré duality}

We recall the Poincare duality and global intersection products of homology classes.

Suppose that $M$ is connected and oriented. Then the pairing

$$
A^{p}(M) \times A_{c}^{m-p}(M) \longrightarrow \mathbb{C} \text { given by }(\omega, \varphi) \mapsto \int_{M} \omega \wedge \varphi
$$

induces the Poincaré duality for a possibly non-compact manifold:

$$
P: H^{p}(M, \mathbb{C}) \simeq H_{\mathrm{dR}}^{p}(M) \stackrel{\sim}{\longrightarrow} H_{c}^{m-p}(M)^{*} \simeq \breve{H}_{m-p}(M, \mathbb{C}) .
$$


In the sequel, we sometimes omit the coefficient $\mathbb{C}$ in homology and cohomology. In fact the Poincaré duality holds with $\mathbb{Z}$ coefficient. Note that $P$ is given by the left cap product with the fundamental class of $M$, the class of the sum of all $m$-simplices in $M$. We also denote $P$ by $P_{M}$ if we wish to make the manifold $M$ under consideration explicit.

In the isomorphism (3.1), the class $[\omega]$ of a closed $p$-form $\omega$ corresponds to the functional on $H_{c}^{m-p}(M)$ given by

$$
[\varphi] \mapsto \int_{M} \omega \wedge \varphi,
$$

or to the class $[C]$ of an $(m-p)$-cycle $C$ such that

$$
\int_{M} \omega \wedge \varphi=\int_{C} \varphi
$$

for any closed form $\varphi$ in $A_{c}^{m-p}(M)$. We call $\omega$ a de Rham representative of $C$.

For two classes $\left[C_{1}\right] \in \breve{H}_{q_{1}}(M)$ and $\left[C_{2}\right] \in \breve{H}_{q_{2}}(M)$, the intersection product $\left[C_{1}\right] \cdot\left[C_{2}\right]$ is defined by

$$
\left[C_{1}\right] \cdot\left[C_{2}\right]:=P\left(P^{-1}\left[C_{1}\right] \smile P^{-1}\left[C_{2}\right]\right) \quad \text { in } \breve{H}_{q_{1}+q_{2}-m}(M),
$$

where $\smile$ denotes the cup product, which corresponds to the exterior product in the first isomorphism in (3.1).

If $M$ is compact and connected, then $\breve{H}_{0}(M, \mathbb{C})=H_{0}(M, \mathbb{C})=\mathbb{C}$. Thus if $q_{1}+q_{2}=m$, $\left[C_{1}\right] \cdot\left[C_{2}\right]$ is a number given by

$$
\left[C_{1}\right] \cdot\left[C_{2}\right]=\int_{M} \omega_{1} \wedge \omega_{2}=\int_{C_{1}} \omega_{2}=(-1)^{q_{1} q_{2}} \int_{C_{2}} \omega_{1},
$$

where $\omega_{1}$ and $\omega_{2}$ are de Rham representatives of $C_{1}$ and $C_{2}$, respectively.

\section{2 Čech-de Rham cohomology}

The Čech-de Rham cohomology is defined for an arbitrary open covering of $M$; however, here we only consider coverings consisting of two open sets. Thus, let $\mathcal{U}=\left\{U_{0}, U_{1}\right\}$ be an open covering of $M$. We set $U_{01}=U_{0} \cap U_{1}$ and define the complex vector space $A^{p}(\mathcal{U})$ as

$$
A^{p}(\mathcal{U}):=A^{p}\left(U_{0}\right) \oplus A^{p}\left(U_{1}\right) \oplus A^{p-1}\left(U_{01}\right) .
$$

An element $\sigma$ in $A^{p}(\mathcal{U})$ is given by a triple $\sigma=\left(\sigma_{0}, \sigma_{1}, \sigma_{01}\right)$ with $\sigma_{i}$ a $p$-form on $U_{i}$, $i=0,1$, and $\sigma_{01}$ a $(p-1)$-form on $U_{01}$. We define an operator $D: A^{p}(\mathcal{U}) \rightarrow A^{p+1}(\mathcal{U})$ by

$$
D \sigma:=\left(d \sigma_{0}, d \sigma_{1},\left.\sigma_{1}\right|_{U_{01}}-\left.\sigma_{0}\right|_{U_{01}}-d \sigma_{01}\right) .
$$

Then we see that $D \circ D=0$ so that we have a complex $\left(A^{*}(\mathcal{U}), D\right)$. The $p$-th $\check{C} e c h-d e$ Rham cohomology of $\mathcal{U}$, denoted by $H_{D}^{p}(\mathcal{U})$, is the $p$-th cohomology of this complex. It is also abbreviated as ČdR cohomology. We denote the class of a cocycle $\sigma$ by $[\sigma]$. It can be shown that the map $A^{p}(M) \rightarrow A^{p}(\mathcal{U})$ given by $\omega \mapsto\left(\left.\omega\right|_{U_{0}},\left.\omega\right|_{U_{1}}, 0\right)$ induces an isomorphism

$$
\alpha: H_{\mathrm{dR}}^{p}(M) \stackrel{\sim}{\longrightarrow} H_{D}^{p}(\mathcal{U}) .
$$

Note that $\alpha^{-1}$ assigns to the class of a ČdR cocycle $\left(\sigma_{0}, \sigma_{1}, \sigma_{01}\right)$ the class of the closed form $\rho_{0} \sigma_{0}+\rho_{1} \sigma_{1}-d \rho_{0} \wedge \sigma_{01}$, where $\left\{\rho_{0}, \rho_{1}\right\}$ is a partition of unity subordinate to $\mathcal{U}$.

Now we could define the cup product for ČdR cochains and describe the Poincaré duality in terms of the $\check{C} d R$ cohomology as in [12] in the case $M$ is compact. However, here we 
proceed as follows. Let $M$ and $\mathcal{U}=\left\{U_{0}, U_{1}\right\}$ be as above. A system of honeycomb cells adapted to $\mathcal{U}$ is a collection $\left\{R_{0}, R_{1}\right\}$ of two submanifolds of $M$ of dimension $m$ with $C^{\infty}$ boundary having the following properties:

(1) $R_{i} \subset U_{i}$ for $i=0,1$,

(2) Int $R_{0} \cap$ Int $R_{1}=\varnothing$ and

(3) $R_{0} \cup R_{1}=M$,

where Int denotes the interior. Suppose $M$ is oriented. Then $R_{0}$ and $R_{1}$ are naturally oriented. Let $R_{01}=R_{0} \cap R_{1}$ with the orientation as the boundary of $R_{0} ; R_{01}=\partial R_{0}$, or equivalently, the orientation opposite to that of the boundary of $R_{1} ; R_{01}=-\partial R_{1}$. We consider the pairing

$$
A^{p}(\mathcal{U}) \times A_{c}^{m-p}(M) \longrightarrow \mathbb{C}
$$

given by

$$
(\sigma, \varphi) \mapsto \int_{R_{0}} \sigma_{0} \wedge \varphi+\int_{R_{1}} \sigma_{1} \wedge \varphi+\int_{R_{01}} \sigma_{01} \wedge \varphi .
$$

Then it induces the Poincaré duality (3.1) through the isomorphism $\alpha$ in (3.5).

\subsection{Relative Čech-de Rham cohomology and Alexander duality}

We introduce the relative Čech-de Rham cohomology and describe the Alexander duality, which is used to define localized intersection products.

Let $S$ be a closed set in $M$. Letting $U_{0}=M \backslash S$ and $U_{1}$ a neighborhood of $S$ in $M$, we consider the covering $\mathcal{U}=\left\{U_{0}, U_{1}\right\}$ of $M$. If we set

$$
A^{p}\left(\mathcal{U}, U_{0}\right)=\left\{\sigma \in A^{p}(\mathcal{U}) \mid \sigma_{0}=0\right\},
$$

we see that $\left(A^{*}\left(\mathcal{U}, U_{0}\right), D\right)$ is a subcomplex of $\left(A^{*}(\mathcal{U}), D\right)$. We denote by $H_{D}^{p}\left(\mathcal{U}, U_{0}\right)$ the $p$-th cohomology of this complex. From the short exact sequence

$$
0 \longrightarrow A^{*}\left(\mathcal{U}, U_{0}\right) \stackrel{j^{*}}{\longrightarrow} A^{*}(\mathcal{U}) \stackrel{\iota^{*}}{\longrightarrow} A^{*}\left(U_{0}\right) \longrightarrow 0,
$$

where $j^{*}$ is the inclusion and $\iota^{*}$ is the homomorphism that assigns $\sigma_{0}$ to $\sigma=\left(\sigma_{0}, \sigma_{1}, \sigma_{01}\right)$, we have the long exact sequence

$$
\cdots \longrightarrow H_{D}^{p-1}(\mathcal{U}) \stackrel{\iota^{*}}{\longrightarrow} H_{\mathrm{dR}}^{p-1}\left(U_{0}\right) \stackrel{\delta^{*}}{\longrightarrow} H_{D}^{p}\left(\mathcal{U}, U_{0}\right) \stackrel{j^{*}}{\longrightarrow} H_{D}^{p}(\mathcal{U}) \stackrel{\iota^{*}}{\longrightarrow} H_{\mathrm{dR}}^{p}\left(U_{0}\right) \longrightarrow \cdots
$$

In the above, $\delta^{*}$ assigns the class $[(0,0,-\theta)]$ to the class of a closed $(p-1)$-form $\theta$ on $U_{0}$. Comparing with the long cohomology exact sequence for the pair $(M, M \backslash S)$, we have a natural isomorphism (see [13] for a precise proof):

$$
H_{D}^{p}\left(\mathcal{U}, U_{0}\right) \simeq H^{p}(M, M \backslash S ; \mathbb{C}) .
$$

We describe the Alexander duality in terms of the relative ČdR cohomology in the case $S$ is compact and admits a regular neighborhood (cf. [12]). Thus, suppose $M$ is oriented and let $\left\{R_{0}, R_{1}\right\}$ be a system of honeycomb cells adapted to $\mathcal{U}$. We assume that $S$ is compact so that we may also assume that $R_{1}$ is compact. Consider the pairing

$$
A^{p}\left(\mathcal{U}, U_{0}\right) \times A^{m-p}\left(U_{1}\right) \longrightarrow \mathbb{C}
$$


given by

$$
(\sigma, \varphi) \mapsto \int_{R_{1}} \sigma_{1} \wedge \varphi+\int_{R_{01}} \sigma_{01} \wedge \varphi
$$

Then it induces the Alexander homomorphism:

$$
A: H^{p}(M, M \backslash S ; \mathbb{C}) \simeq H_{D}^{p}\left(\mathcal{U}, U_{0}\right) \longrightarrow H_{\mathrm{dR}}^{m-p}\left(U_{1}\right)^{*} \simeq H_{m-p}\left(U_{1}, \mathbb{C}\right) .
$$

The homomorphism (3.9) depends on $U_{1}$ and is not an isomorphism in general. Here we consider the following hypothesis :

(*) there exists a triangulation of $M$ such that $S$ is (the polyhedron of) a subcomplex.

In this case, we also say that the triangulation is compatible with $S$.

Remark 3.1 The triangulation above may simply be $C^{0}$ to have the Alexander duality (3.10) below. We mainly consider $C^{\infty}$ triangulations in order that, in an expression as (3.12), the integral in the right-hand side makes sense. The hypothesis is certainly satisfied if $S$ is the support of a chain. It is also satisfied if $S$ is a $C^{\infty}$ submanifold of $M$.

Another case of interest where the following arguments go through is that $S$ is a subanalytic set in a real analytic manifold $M$, as in this case, there is a subanalytic triangulation of $M$ compatible with $S$ (cf. [11]) so that the integration makes sense, see Example 4.13 below.

Under the hypothesis (*), we may take as $U_{1}$ a regular neighborhood of $S$ so that there is a deformation retract $U_{1} \rightarrow S$. We then have $H_{m-p}\left(U_{1}\right) \simeq H_{m-p}(S)$ and (3.8) induces the Alexander duality:

$$
A: H^{p}(M, M \backslash S ; \mathbb{C}) \simeq H_{D}^{p}\left(\mathcal{U}, U_{0}\right) \stackrel{\sim}{\longrightarrow} H_{\mathrm{dR}}^{m-p}\left(U_{1}\right)^{*} \simeq H_{m-p}(S, \mathbb{C}) .
$$

Note that $A$ is also given by the left cap product with the fundamental class $M$. We also denote $A$ by $A_{M, S}$ if we wish to make the pair $(M, S)$ explicit.

In the isomorphism (3.10), the class $[\sigma]$ of a $p$-cochain $\sigma$ corresponds to the functional on $H_{\mathrm{dR}}^{m-p}\left(U_{1}\right)$ given by

$$
\left[\varphi_{1}\right] \mapsto \int_{R_{1}} \sigma_{1} \wedge \varphi_{1}+\int_{R_{01}} \sigma_{01} \wedge \varphi_{1},
$$

or to the class $[C]$ of an $(m-p)$-cycle $C$ in $S$ such that

$$
\int_{R_{1}} \sigma_{1} \wedge \varphi_{1}+\int_{R_{01}} \sigma_{01} \wedge \varphi_{1}=\int_{C} \varphi_{1}
$$

for any closed form $\varphi_{1}$ in $A^{m-p}\left(U_{1}\right)$.

Denoting by $i: S \hookrightarrow M$ the inclusion, we have the following commutative diagram:

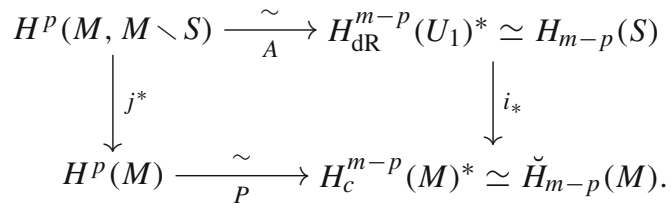

Remark 3.2 1. In the above, the homomorphism $i_{*}: H_{m-p}(S) \rightarrow \breve{H}_{m-p}(M)$ is the one naturally induced from $i$, while $i_{*}: H_{\mathrm{dR}}^{m-p}\left(U_{1}\right)^{*} \rightarrow H_{c}^{m-p}(M)^{*}$ is described as follows. 
For any functional $F_{1}$ on $H_{\mathrm{dR}}^{m-p}\left(U_{1}\right)$, there is a corresponding cycle $C$ in $S$ and $i_{*} F_{1}$ is given by

$$
i_{*} F_{1}[\varphi]=\int_{C} \varphi \quad \text { for }[\varphi] \in H_{c}^{m-p}(M) .
$$

Alternatively, if $\left(0, \sigma_{1}, \sigma_{01}\right)$ is a $\check{C} \mathrm{dR}$ representative of $A^{-1} F_{1}$, then

$$
i_{*} F_{1}[\varphi]=\int_{R_{1}} \sigma_{1} \wedge \varphi+\int_{R_{01}} \sigma_{01} \wedge \varphi .
$$

2. For a closed set $S$ (which may not be compact) in $M$ satisfying (*), we may define the Alexander isomorphism

$$
A: H^{p}(M, M \backslash S) \stackrel{\sim}{\longrightarrow} \breve{H}_{m-p}(S)
$$

via combinatorial topology (cf. [3]).

Let $S_{1}$ and $S_{2}$ be compact sets in $M$ satisfying (*) and set $S=S_{1} \cap S_{2}$. Let $A_{1}, A_{2}$ and $A$ denote the Alexander isomorphisms for $\left(M, S_{1}\right),\left(M, S_{2}\right)$ and $(M, S)$, respectively. For two classes $c_{1} \in H_{q_{1}}\left(S_{1}\right)$ and $c_{2} \in H_{q_{2}}\left(S_{2}\right)$, the localized intersection product $\left(c_{1} \cdot c_{2}\right)_{S}$ is defined by

$$
\left(c_{1} \cdot c_{2}\right)_{S}:=A\left(A_{1}^{-1} c_{1} \smile A_{2}^{-1} c_{2}\right) \quad \text { in } H_{q_{1}+q_{2}-m}(S),
$$

where $\smile$ denotes the cup product

$$
H^{m-q_{1}}\left(M, M \backslash S_{1}\right) \times H^{m-q_{2}}\left(M, M \backslash S_{2}\right) \stackrel{\smile}{\longrightarrow} H^{2 m-q_{1}-q_{2}}(M, M \backslash S) .
$$

Letting $i_{1}: S_{1} \hookrightarrow M, i_{2}: S_{2} \hookrightarrow M$ and $i: S \hookrightarrow M$ be the inclusions, from (3.13), we see that the definitions (3.4) and (3.14) are consistent in the sense that

$$
i_{*}\left(c_{1} \cdot c_{2}\right)_{S}=\left(i_{1}\right)_{*} c_{1} \cdot\left(i_{2}\right)_{*} c_{2} .
$$

\subsection{Thom class}

We list [9] as a general reference for the Thom isomorphism and the Thom class of a real vector bundle. In general, they are defined in cohomology with $\mathbb{Z}_{2}$ coefficients, while for an oriented vector bundle, they can be defined in cohomology with $\mathbb{Z}$ coefficients. They can also be described in terms of differential forms, in which case the cohomology involved is with $\mathbb{C}$ coefficients. This is done in [2] using cohomology with compact support in the vertical direction. Here we use Čech-de Rham cohomology instead as in [12]. This way we can express relevant local informations more explicitly.

In this subsection, we sometimes omit the coefficient $\mathbb{C}$ in homology and cohomology. In fact the isomorphisms we consider below can be defined from combinatorial viewpoint in homology and cohomology with $\mathbb{Z}$ coefficient (cf. [3], also [13]).

(a) Thom class of an oriented real vector bundle Let $\pi: E \rightarrow M$ be an oriented real vector bundle of rank $k$. We identify $M$ with the image of the zero section. Then we have the Thom isomorphism

$$
T_{E}: H^{p}(M, \mathbb{C}) \stackrel{\sim}{\longrightarrow} H^{p+k}(E, E \backslash M ; \mathbb{C}),
$$

whose inverse is given by the integration along the fiber of $\pi$ (see [12, Ch.II, 5]).

The Thom class $\Psi_{E}$ of $E$, which is in $H^{k}(E, E \backslash M)$, is the image of the constant function 1 in $H^{0}(M, \mathbb{C})$ by $T_{E}$. Note that $T_{E}$ is given by the cup product with $\Psi_{E}$. Let $W_{0}=E \backslash M$ 
and $W_{1}$ a neighborhood of $M$ in $E$ and consider the covering $\mathcal{W}=\left\{W_{0}, W_{1}\right\}$ of $E$. We refer to [12, Ch.II, Proposition 5.7] for an explicit expression of a ČdR cocycle representing $\Psi_{E}$ in the isomorphism $H^{k}(E, E \backslash M) \simeq H_{D}^{k}\left(\mathcal{W}, W_{0}\right)$. In particular, suppose $E$ is trivial on an open set $U$ of $M$. Then, setting $\left.A^{k}\left(\mathcal{W}, W_{0}\right)\right|_{U}=A^{k}\left(\mathcal{W}^{\prime}, W_{0}^{\prime}\right)$ with $W_{i}^{\prime}=W_{i} \cap \pi^{-1}(U)$, we have (cf. [12, Ch.III, Lemma 1.4]):

Proposition 3.3 Suppose $E$ is trivial on an open set $U$ of $M ;\left.E\right|_{U} \simeq \mathbb{R}^{k} \times U$ and let $\rho:\left.E\right|_{U} \rightarrow \mathbb{R}^{k}$ denote the projection on to the fiber direction. Then $\Psi_{\left.E\right|_{U}}$ is represented by a cocycle in $\left.A^{k}\left(\mathcal{W}, W_{0}\right)\right|_{U}$ of the form

$$
\left(0,0,-\rho^{*} \psi_{k}\right),
$$

where $\psi_{k}$ is an angular form on $\mathbb{R}^{k} \backslash\{0\}$, i.e., a closed $(k-1)$-form with $\int_{S^{k-1}} \psi_{k}=1$.

Suppose $M$ is compact and oriented. We orient the total space $E$ so that if $\xi=\left(\xi_{1}, \ldots, \xi_{k}\right)$ is a positive fiber coordinate system of $E$ and if $x=\left(x_{1}, \ldots, x_{m}\right)$ is a positive coordinate system on $M$, then $(\xi, x)$ is a positive coordinate system on $E$. We then have the commutative diagram :

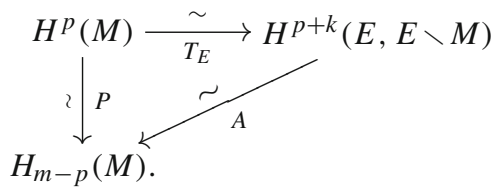

(b) Thom class of a submanifold Let $W$ be an oriented $C^{\infty}$ manifold of dimension $m^{\prime}$ and $M$ a compact and oriented submanifold of $W$ of dimension $m$. Set $k=m^{\prime}-m$. In view of (3.15), we define the Thom isomorphism $T_{M}: H^{p}(M) \stackrel{\sim}{\rightarrow} H^{p+k}(W, W \backslash M)$ so that the following diagram becomes commutative (cf. [3]):

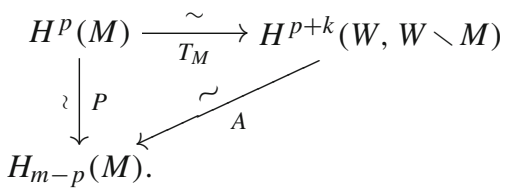

Then the Thom class $\Psi_{M}$ of $M$ is defined by

$$
\Psi_{M}=: T_{M}(1)=A^{-1}(M) \quad \text { in } H^{k}(W, W \backslash M) .
$$

Let $N_{M} \rightarrow M$ denote the normal bundle of $M$ in $W$. Suppose the orientation of $M$ is compatible with that of $W$ in the sense that $N_{M}$ is orientable. We orient $N_{M}$ as follows. Namely, if $\left(x_{1}, \ldots, x_{k}, \ldots, x_{m^{\prime}}\right)$ is a positive coordinate system on $W$ such that $M$ is given by $x_{1}=\cdots=x_{k}=0$ and that $\left(x_{k+1}, \ldots, x_{m^{\prime}}\right)$ is a positive coordinate system of $M$, then the vectors $\left(\frac{\partial}{\partial x_{1}}, \ldots, \frac{\partial}{\partial x_{k}}\right)$ determine a positive frame of $N_{M}$. By the tubular neighborhood theorem, there is a neighborhood $W_{1}$ of $M$ in $W$ and an orientation preserving diffeomorphism of $W_{1}$ onto a neighborhood of the zero section of $N_{M}$, which is identified with $M$. By excision, we have

$$
H^{k}(W, W \backslash M) \simeq H^{k}\left(W_{1}, W_{1} \backslash M\right) \simeq H^{k}\left(N_{M}, N_{M} \backslash M\right)
$$

and in the above isomorphisms, the Thom class $\Psi_{M}$ of $M$ corresponds to the Thom class $\Psi_{N_{M}}$ of the vector bundle $N_{M}$. 
Remark 3.4 More generally, for a pseudo-manifold $M$ in $W$, we may define the Poincaré homomorphism $P: H^{p}(M) \longrightarrow \breve{H}_{m-p}(M)$. Thus, we have the Thom homomorphism and the Thom class $\Psi_{M}$ of $M$ (cf. [3]).

(c) Thom class of a cycle Let $C$ be a finite $\left(m^{\prime}-p\right)$-cycle in $W$ and $\tilde{S}$ its support. We may define the Thom class $\Psi_{C}$ of $C$ by

$$
\Psi_{C}:=A^{-1}(C)
$$

where $A$ is the Alexander isomorphism (3.10) for the pair $(W, \tilde{S})$.

\section{Localized intersection of currents}

\subsection{Thom class of a current}

Let $W$ be an oriented $C^{\infty}$ manifold of dimension $m^{\prime}$. Recall that a $p$-current $T$ on $W$ is a continuous linear functional on the space $A_{c}^{m^{\prime}-p}(W)$. We use the notation

$$
T(\varphi)=\langle T, \varphi\rangle, \quad \varphi \in A_{c}^{m^{\prime}-p}(W) .
$$

Let $\mathcal{D}^{p}(W)$ denote the space of $p$-currents on $W$. The differential $d: \mathcal{D}^{p}(W) \rightarrow \mathcal{D}^{p+1}(W)$ is defined by

$$
\langle d T, \varphi\rangle=(-1)^{p+1}\langle T, d \varphi\rangle, \quad \varphi \in A_{c}^{m^{\prime}-p-1}(W) .
$$

Then $\left(\mathcal{D}^{*}(W), d\right)$ forms a complex, whose $p$-th cohomology is denoted by $H^{p}\left(\mathcal{D}^{*}(W)\right)$. For a closed $p$-current $T$, we denote by $[T]$ its cohomology class.

A form $\omega$ in $A^{p}(W)$ may be naturally thought of as a $p$-current $T_{\omega}$ by

$$
\left\langle T_{\omega}, \varphi\right\rangle=\int_{W} \omega \wedge \varphi, \quad \varphi \in A_{c}^{m^{\prime}-p}(W) .
$$

If $\omega$ is closed, then $T_{\omega}$ is closed and the assignment $\omega \mapsto T_{\omega}$ induces an isomorphism

$$
\beta: H_{\mathrm{dR}}^{p}(W) \stackrel{\sim}{\longrightarrow} H^{p}\left(\mathcal{D}^{*}(W)\right) .
$$

A Čech-de Rham cochain $\sigma$ on a covering $\mathcal{W}$ of $W$ may be also thought of as a current $T_{\sigma}$ via integration given as (3.6). If $D \sigma=0$, then $T_{\sigma}$ is closed and the assignment $\sigma \mapsto T_{\sigma}$ induces the isomorphism $\beta \circ \alpha^{-1}: H_{D}^{p}(\mathcal{W}) \stackrel{\sim}{\rightarrow} H^{p}\left(\mathcal{D}^{*}(W)\right)$.

Also an $\left(m^{\prime}-p\right)$-chain $C$ may be thought of as a $p$-current $T_{C}$ by

$$
\left\langle T_{C}, \varphi\right\rangle=\int_{C} \varphi, \quad \varphi \in A_{c}^{m^{\prime}-p}(W) .
$$

If $C$ is a cycle, $T_{C}$ is closed and the assignment $C \mapsto T_{C}$ induces an isomorphism

$$
\gamma: \breve{H}_{m-p}(W) \stackrel{\sim}{\longrightarrow} H^{p}\left(\mathcal{D}^{*}(W)\right) .
$$

By (3.3), we have $\gamma \circ P=\beta$. Thus for any closed current $T$ on $W$, there exist a closed $p$-form $\omega$, a ČdR cocycle $\sigma$ and an $\left(m^{\prime}-p\right)$-cycle $C$ such that

$$
[T]=\left[T_{\omega}\right]=\left[T_{\sigma}\right]=\left[T_{C}\right] .
$$

We call $\omega, \sigma$ and $C$, respectively, de Rham, $\check{C} \mathrm{dR}$ and cycle representatives of $T$. 
If $U$ is an open set of $W$, there is a natural inclusion $A_{c}^{*}(U) \hookrightarrow A_{c}^{*}(W)$, given by extension by zero, so that we may consider the restriction $\left.T\right|_{U}$ to $U$ of a current $T$ on $W$. The support $\operatorname{supp}(T)$ of $T$ is the smallest closed subset of $W$ such that $\left.T\right|_{W \backslash \operatorname{supp}(T)}=0$.

Now we consider the localization problem of currents. Thus, let $\tilde{S}$ be a closed set in $W$. Let $W_{0}=W \backslash \tilde{S}$ and $W_{1}$ a neighborhood of $\tilde{S}$ in $W$ and consider the covering $\mathcal{W}=\left\{W_{0}, W_{1}\right\}$ of $W$. We have the commutative diagram with exact row (cf. (3.7)):

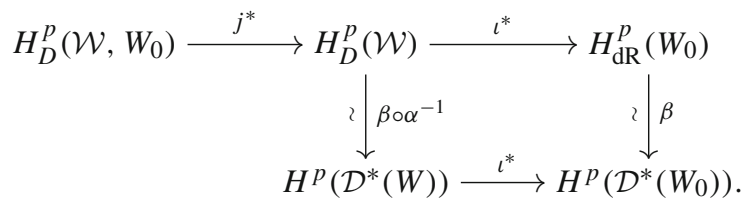

Suppose $T$ is a closed $p$-current on $W$ such that $\iota^{*}[T]=0$, i.e., $\left[\left.T\right|_{W_{0}}\right]=0$. Then there is a class $\Psi_{T}$ in $H_{D}^{p}\left(\mathcal{W}, W_{0}\right)$ such that $[T]=j^{*} \Psi_{T}$. We then say that $T$ is localized at $\tilde{S}$ and call $\Psi_{T}$ a Thom class of $T$ along $\tilde{S}$. Here some comments are in order:

1. Any closed current $T$ is localized at $\operatorname{supp}(T)$ in the above sense. It is also localized at the support of a cycle representative of $T$. Thus, the set $\tilde{S}$ as above may be different from $\operatorname{supp}(T)$, see Example 4.3 below.

2. The class $\Psi_{T}$ is not uniquely determined, as $j^{*}$ is not injective in general; however in some cases, there is a natural choice of $\Psi_{T}$, see Examples 4.1 and 4.5 below.

In the above situation, suppose $\tilde{S}$ is a compact set satisfying (*). Let $\left(0, \psi_{1}, \psi_{01}\right)$ be a ČdR representative of $\Psi_{T}$ and $\left\{\tilde{R}_{0}, \tilde{R}_{1}\right\}$ a system of honeycomb cells adapted to $\mathcal{W}$. Then, from the commutativity of the diagram obtained by replacing $M$ and $\mathcal{U}$ by $W$ and $\mathcal{W}$ in (3.13) (see also Remark 3.2), for any closed form $\varphi$ in $A_{c}^{m^{\prime}-p}(W)$, we have

$$
\langle T, \varphi\rangle=\int_{\tilde{R}_{1}} \psi_{1} \wedge \varphi+\int_{\tilde{R}_{01}} \psi_{01} \wedge \varphi .
$$

Thus, the value of $T$ is "concentrated" near $\tilde{S}$ and is explicitly given by the above. Also note that the right-hand side does not depend on the choice of $\Psi_{T}$.

Example 4.1 Let $C$ be a finite $\left(m^{\prime}-p\right)$-cycle in $W$. Then $T_{C}$ is localized at $\tilde{S}=|C|$ and $\Psi_{C}$ is a natural choice for $\Psi_{T_{C}}$. In particular, if $C=M$ is a compact oriented submanifold of codimension $p$, then the Thom class $\Psi_{M}$ of $M$ is a natural choice for $\Psi_{T_{M}}$.

We show that, starting from a closed $1 p$-form $\omega$ such that $\left[T_{\omega}\right]=[T]$, there is a natural way of constructing such a class. Thus, let $\tilde{S}$ be a closed set of $W$ and let $\mathcal{W}=\left\{W_{0}, W_{1}\right\}$ be as before.

Proposition 4.2 Let $T$ be a closed p-current on $W$ such that $\left[\left.T\right|_{W_{0}}\right]=0$ and $\omega$ a de Rham representative of $T$. Then there exists a Thom class $\Psi_{T}$ which is represented by a $\check{C} d R$ cocycle of the form $(0, \omega,-\psi)$ with $\psi$ a $(p-1)$-form on $W_{01}$ satisfying $\omega=-d \psi$ on $W_{01}$.

Proof Just to make sure we denote the restrictions of forms explicitly. From the assumption, there exists a $(p-1)$-form $\psi$ on $W_{0}$ such that $\mathrm{d} \psi=-\left.\omega\right|_{W_{0}}$. Hence, the cocycle $\left(\left.\omega\right|_{W_{0}},\left.\omega\right|_{W_{1}}, 0\right)$ is cohomologous as a ČdR cocycle to $\left(0,\left.\omega\right|_{W_{1}},-\left.\psi\right|_{W_{01}}\right)$ since

$$
\left(0,\left.\omega\right|_{W_{1}},-\left.\psi\right|_{W_{01}}\right)-\left(\left.\omega\right|_{W_{0}},\left.\omega\right|_{W_{1}}, 0\right)=\left(\mathrm{d} \psi, 0,-\left.\psi\right|_{W_{01}}\right)=D(\psi, 0,0) .
$$

Thus, the class $\Psi_{T}=\left[\left(0,\left.\omega\right|_{W_{1}},-\left.\psi\right|_{W_{01}}\right)\right]$ satisfies $[T]=j^{*} \Psi_{T}$. 
In this case, if $\tilde{S}$ is a compact set satisfying (*), (4.3) is written as

$$
\int_{W} \omega \wedge \varphi=\int_{\tilde{R}_{1}} \omega \wedge \varphi-\int_{\tilde{R}_{01}} \psi \wedge \varphi .
$$

Thus, the value of the integral away from $\tilde{R}_{1}$ is cut off and is compensated by an integral on $\tilde{R}_{01}$.

Example 4.3 Let $C$ be an $\left(m^{\prime}-p\right)$-cycle in $W$ and $\omega$ a de Rham representative of $C$. Let $\tilde{S}$ be the support of $C$ and set $W_{0}=W \backslash \tilde{S}$. Then $T_{\omega}$ is localized at $\tilde{S}$ as $\left[T_{\omega}\right]=\left[T_{C}\right]$, although we do not have any precise information about $\operatorname{supp}\left(T_{\omega}\right)$. Its Thom class $\Psi_{T_{\omega}}$ along $\tilde{S}$ is represented by a $\breve{C} \mathrm{dR}$ cocycle of the form $(0, \omega,-\psi)$.

Remark 4.4 Let $C, \omega$ and $\tilde{S}$ be as in Example 4.3. Then there is a $(p-1)$-current $R$ such that

$$
T_{C}-T_{\omega}=d R
$$

We may think of $R$ as the current defined by a $(p-1)$-form $\psi$ on $W \backslash \tilde{S}$ that can be extended as a locally integrable $L^{1}$ form on $W$ and with $d \psi=-\omega$ on $W \backslash \tilde{S}$. The equation above becomes then

$$
\mathrm{d} T_{\psi}-T_{\mathrm{d} \psi}=T_{C},
$$

which is a residue formula (cf. $[5$, Ch.3,1]), and the identity

$$
D(\psi, 0,0)+(\omega, \omega, 0)=(0, \omega,-\psi)
$$

may be thought of as the corresponding expression in terms of $\check{C} \mathrm{dR}$ cochains.

Example 4.5 Let $\pi: E \rightarrow W$ be a $C^{\infty}$ complex vector bundle of rank $r$ and $\nabla$ a connection for $E$. For $q=0, \ldots, r$, we have the $q$-th Chern form $c_{q}(\nabla)$, which is a closed $2 q$-form defining the $q$-th Chern class $c_{q}(E)$ in $H_{\mathrm{dR}}^{2 q}(W)$. We call $T_{c_{q}(\nabla)}$ the $q$-th Chern current associated with $\nabla$. Suppose $E$ admits $\ell$ sections $s=\left(s_{1}, \ldots, s_{\ell}\right)$ that are linearly independent on the complement of a closed set $\tilde{S} \subset W$. Then we see that $T_{c_{q}(\nabla)}$ is localized at $\tilde{S}$ and there is a natural way of choosing a Thom class along $\tilde{S}$ for $q=r-\ell+1, \ldots, r$.

For this, we take an $s$-trivial connection $\nabla_{0}$ for $E$ on $W_{0}=W \backslash \tilde{S}$, i.e., a connection satisfying $\nabla_{0} s_{i}=0$ for $i=1, \ldots, \ell$. Denoting by $c_{q}\left(\nabla_{0}, \nabla\right)$ the Bott difference form (cf. $[1,12])$, we have $\left.c_{q}(\nabla)\right|_{W_{0}}-c_{q}\left(\nabla_{0}\right)=d c_{q}\left(\nabla_{0}, \nabla\right)$. Since the connection $\nabla_{0}$ is $s$-trivial, it follows that $c_{q}\left(\nabla_{0}\right)=0$ so that $\left.c_{q}(\nabla)\right|_{W_{0}}$ is exact for $q=r-\ell+1, \ldots, r$. Hence, the Chern current $T_{c_{q}(\nabla)}$ localizes at $\tilde{S}$. As its Thom class along $\tilde{S}$, we may take the class $c_{q}(E, s)$ in $H^{2 q}(W, W \backslash \tilde{S}) \simeq H_{D}^{2 q}\left(\mathcal{W}, W_{0}\right)$ represented by the cocycle (cf. Proposition 4.2):

$$
\left(0,\left.c_{q}(\nabla)\right|_{W_{1}}, c_{q}\left(\nabla_{0}, \nabla\right)\right) .
$$

This class does not depend on the choice of $\nabla$ or $\nabla_{0}$ (cf. [12, Ch.III, Lemma 3.1]) and is a natural choice of Thom class for $T_{c_{q}(\nabla)}$. It is the localization of $c_{q}(E)$ at $\tilde{S}$ by $s$.

The Thom class of a complex vector bundle as a real oriented bundle may be expressed in this manner (cf. [12, Ch.III, Theorem 4.4]). 


\subsection{Localized intersection of currents}

Let $W$ be an oriented $C^{\infty}$ manifold of dimension $m^{\prime}$ as before. For closed currents $T_{1}$ and $T_{2}$, it is possible to define the intersection product $T_{1} \cdot T_{2}$ in the homology of $W$ using the isomorphism $\beta$ and the Poincaré duality [cf. (4.1), (3.4)]. Also if $T_{1}$ and $T_{2}$ are localized at compact sets $\tilde{S}_{1}$ and $\tilde{S}_{2}$ satisfying (*), we may define the localized intersection $\left(T_{1} \cdot T_{2}\right)_{\tilde{S}}$ in the homology of $\tilde{S}=\tilde{S}_{1} \cap \tilde{S}_{2}$ using Thom classes $\Psi_{T_{1}}$ and $\Psi_{T_{2}}$ and the Alexander duality [cf. (3.14)].

Here we consider the case $T_{1}=T_{M}$ with $M$ a compact oriented submanifold of dimension $m$ in $W$ and obtain a residue theorem on $M$. In the sequel, we take the Thom class $\Psi_{M}$ of $M$ [cf. Sect. 3.4 (b)] as $\Psi_{T_{M}}$ and set $k=m^{\prime}-m$. Recall that by the Alexander isomorphism

$$
A_{W, M}: H^{k}(W, W \backslash M) \stackrel{\sim}{\longrightarrow} H_{m}(M),
$$

the class $\Psi_{M}$ corresponds to the fundamental class $M$. Let $i: M \hookrightarrow W$ denote the inclusion. First localization Let $c$ be a class in $\breve{H}_{m^{\prime}-p}(W)$. Recall that we have the intersection product $(M \cdot c)_{M}$ localized at $M$ [cf. (3.14)], which is a class in $H_{m-p}(M)$ defined as $A_{W, M}\left(\Psi_{M} \smile\right.$ $\left.P_{W}^{-1} c\right)$. We denote it by $M \cdot c$ :

$$
M \cdot c:=(M \cdot c)_{M} .
$$

It is sent to $[M] \cdot c$ by $i_{*}: H_{m-p}(M) \rightarrow \breve{H}_{m-p}(W)$.

Second localization Let $\tilde{S}$ be a compact set of $W$ satisfying (*). We have the Alexander isomorphism

$$
A_{W, \tilde{S}}: H^{p}(W, W \backslash \tilde{S}) \stackrel{\sim}{\longrightarrow} H_{m^{\prime}-p}(\tilde{S}) .
$$

We set $S=\tilde{S} \cap M$ and suppose it also satisfies (*). For a class $c$ in $H_{m^{\prime}-p}(\tilde{S})$, we have the class $(M \cdot c)_{S}$ in $H_{m-p}(S)$ [cf. (3.14)].

Proposition 4.6 The following diagrams are commutative:
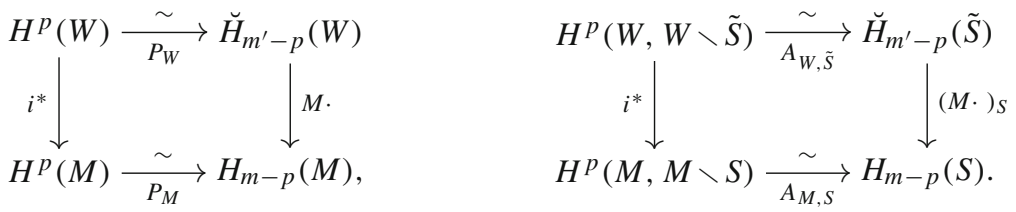

Proof We prove the commutativity of the second diagram, the proof for the first one being similar. We have the cup product followed by the Alexander isomorphism:

$$
H^{k}(W, W \backslash M) \times H^{p}(W, W \backslash \tilde{S}) \stackrel{\smile}{\longrightarrow} H^{k+p}(W, W \backslash S) \stackrel{A_{W, S}}{\longrightarrow} H_{m-p}(S) .
$$

Nothing that the Alexander isomorphism is given by the left cap product with the fundamental class and using properties of cap and cup products, we have, for a class $u$ in $H^{p}(W, W \backslash \tilde{S})$,

$$
A_{M, S}\left(i^{*} u\right)=A_{W, S}\left(\Psi_{M} \smile u\right)=\left(M \cdot A_{W, \tilde{S}} u\right)_{S} .
$$

In view of the above, we define intersection products in a more general situation where $M$ is not necessarily a submanifold of $W$ : 
Definition 4.7 Let $W$ and $M$ be oriented $C^{\infty}$ manifolds of dimensions $m^{\prime}$ and $m$, respectively, and $F: M \rightarrow W$ a $C^{\infty}$ map. We define the intersection product $M \cdot_{F}$ so that the first diagram below is commutative. Also, for a compact set $\tilde{S}$ satisfying (*) in $W$, we set $S=F^{-1}(\tilde{S})$ and suppose $S$ is compact and satisfy $(*)$. We then define the localized intersection product $\left(M \cdot_{F}\right)_{S}$ so that the second diagram is commutative:
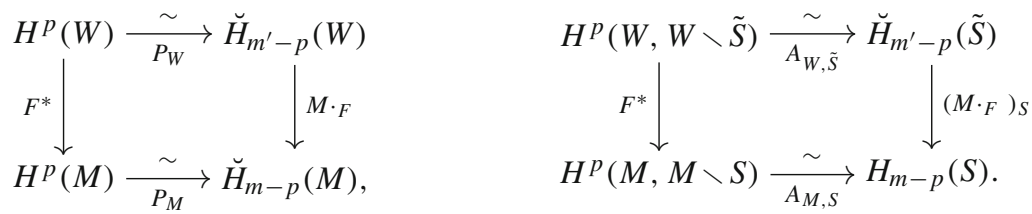

Remark 4.81 . Let $M$ be a submanifold of $W$ and $i: M \hookrightarrow W$ the inclusion. If $M$ is compact, $M \cdot_{i}$ is the product $M \cdot$ defined before. We may also define the product $M \cdot$ as $M \cdot_{i}$ in the case $M$ is not compact.

2. The products as above are defined in the algebraic category in [4].

For a closed $p$-current $T$ on $W$, we define

$$
M \cdot{ }_{F} T:=M \cdot{ }_{F} P \beta^{-1}[T] .
$$

Suppose $T$ is localized at $\tilde{S}$. Then taking a Thom class $\Psi_{T}$ of $T$ along $\tilde{S}$, we define the residue of $\Psi_{T}$ on $M$ at $S$ by

$$
\operatorname{Res}\left(F^{*} \Psi_{T}, S\right):=\left(M \cdot_{F} A\left(\Psi_{T}\right)\right)_{S} .
$$

Suppose $S$ has a finite number of connected components $\left(S_{\lambda}\right)_{\lambda}$. Then we have a decomposition $H_{m-p}(S)=\bigoplus_{\lambda} H_{m-p}\left(S_{\lambda}\right)$ and accordingly $\operatorname{Res}\left(F^{*} \Psi_{T}, S\right)$ determines a class in $H_{m-p}\left(S_{\lambda}\right)$, which is denoted by $\operatorname{Res}\left(F^{*} \Psi_{T}, S_{\lambda}\right)$. We can state the following general residue theorem, which follows from the commutativity of the diagram (3.13):

Theorem 4.9 Let $W$ and $M$ be oriented $C^{\infty}$ manifolds of dimensions $m^{\prime}$ and $m$, respectively, and $F: M \rightarrow W$ a $C^{\infty}$ map. Let $T$ be a closed $p$-current on $W$ such that $\left[\left.T\right|_{W \backslash \tilde{S}}\right]=0$ for some compact subset $\tilde{S}$ satisfying (*) in $W$. Suppose that $S=F^{-1} \tilde{S}$ is compact, satisfies (*) and has a finite number of connected components $\left(S_{\lambda}\right)_{\lambda}$. Then

(1) For each $\lambda$, we have a class $\operatorname{Res}\left(F^{*} \Psi_{T}, S_{\lambda}\right)$ in $H_{m-p}\left(S_{\lambda}\right)$.

(2) We have the "residue formula":

$$
M \cdot{ }_{F} T=\sum_{\lambda}\left(i_{\lambda}\right)_{*} \operatorname{Res}\left(F^{*} \Psi_{T}, S_{\lambda}\right) \quad \text { in } \breve{H}_{m-p}(M),
$$

where $i_{\lambda}: S_{\lambda} \hookrightarrow M$ denotes the inclusion.

We may express $M \cdot_{F} T$ and $\operatorname{Res}\left(F^{*} \Psi_{T}, S_{\lambda}\right)$ as follows. Let $T$ be a closed $p$-current on $W$ and $\omega$ a de Rham representative of $T$. From (3.2) and (3.3), we have

Proposition 4.10 (1) The intersection product $M \cdot_{F} T$ in $\breve{H}_{m-p}(M)$ is represented by a cycle $C$ such that

$$
\int_{M} F^{*} \omega \wedge \varphi=\int_{C} \varphi
$$

for any closed form $\varphi$ in $A_{c}^{m-p}(M)$. 
(2) In the isomorphism $\breve{H}_{m-p}(M) \simeq H_{c}^{m-p}(M)^{*}, M \cdot{ }_{F} T$ corresponds to the functional on $H_{c}^{p}(M)$ that assigns to $[\varphi]$ the left- hand side above.

(3) In particular, if $p=m$ and if $M$ is compact, $M \cdot_{F} T$ is a number given by

$$
M \cdot{ }_{F} T=\int_{M} F^{*} \omega .
$$

Suppose $T$ satisfies the conditions in Theorem 4.9. Let $W_{0}=W \backslash \tilde{S}$ and $W_{1}$ a neighborhood of $\tilde{S}$ and consider the covering $\mathcal{W}=\left\{W_{0}, W_{1}\right\}$. Let $\Psi_{T}$ be represented by a ČdR cocycle $\left(0, \psi_{1}, \psi_{01}\right)$ in $A^{p}\left(\mathcal{W}, W_{0}\right)$. For each $\lambda$, we take a regular neighborhood $U_{\lambda}$ of $S_{\lambda}$ in $M$ such that $F\left(U_{\lambda}\right) \subset W_{1}$ and that $U_{\lambda} \cap U_{\mu}=\emptyset$ if $\lambda \neq \mu$. For each $\lambda$, we take a compact submanifold $R_{\lambda}$ of dimension $m$ with $C^{\infty}$ boundary in $U_{\lambda}$, containing $S_{\lambda}$ in its interior. From (3.11) and (3.12), we have

Proposition 4.11 (1) The residue $\operatorname{Res}\left(F^{*} \Psi_{T}, S_{\lambda}\right)$ in $H_{m-p}\left(S_{\lambda}\right)$ is represented by a cycle $C$ such that

$$
\int_{R_{\lambda}} F^{*} \psi_{1} \wedge \varphi+\int_{R_{0 \lambda}} F^{*} \psi_{01} \wedge \varphi=\int_{C} \varphi
$$

for any closed form $\varphi$ in $A^{m-p}\left(U_{\lambda}\right)$.

(2) In the isomorphism $H_{m-p}\left(S_{\lambda}\right) \simeq H_{\mathrm{dR}}^{m-p}\left(U_{\lambda}\right)^{*}, \operatorname{Res}\left(F^{*} \Psi_{T}, S_{\lambda}\right)$ corresponds to the functional on $H_{\mathrm{dR}}^{m-p}\left(U_{\lambda}\right)$ that assigns to $[\varphi]$ the left-hand side above.

(3) In particular, if $p=m$, the residue is a number given by

$$
\operatorname{Res}\left(F^{*} \Psi_{T}, S_{\lambda}\right)=\int_{R_{\lambda}} F^{*} \psi_{1}+\int_{R_{0 \lambda}} F^{*} \psi_{01} .
$$

Example 4.12 Let $C$ be a finite $\left(m^{\prime}-p\right)$-cycle on $W, \tilde{S}=|C|$ and $S=F^{-1} \tilde{S}$. We take $\Psi_{C}$ as $\Psi_{T_{C}}$. Then

$$
M \cdot{ }_{F} T_{C}=M \cdot{ }_{F}[C], \quad \operatorname{Res}\left(F^{*} \Psi_{C}, S_{\lambda}\right)=\left(M \cdot{ }_{F} C\right)_{S_{\lambda}}
$$

and the residue formula becomes

$$
M \cdot_{F}[C]=\sum_{\lambda}\left(i_{\lambda}\right)_{*}\left(M \cdot_{F} C\right)_{S_{\lambda}} \quad \text { in } \breve{H}_{m-p}(M) .
$$

In particular, if $M$ is compact and $p=m$,

$$
M \cdot{ }_{F}[C]=\sum_{\lambda}\left(M \cdot{ }_{F} C\right)_{S_{\lambda}} .
$$

Let $\omega$ be a de Rham representative of $C$. Then $T_{\omega}$ is localized at $\tilde{S}$. As $\Psi_{T_{\omega}}$, we may take the class represented by a cocycle of the form $(0, \omega,-\psi)$ (cf. Example 4.3). As a homology class, $M \cdot_{F} T_{\omega}=M \cdot{ }_{F}[C]$. As a functional, it is given as in Proposition 4.10. Also $\operatorname{Res}\left(F^{*} \Psi_{T_{\omega}}, S_{\lambda}\right)$ is a functional given as in Proposition 4.11 with $\psi_{1}=\omega$ and $\psi_{01}=-\psi$.

See Propositions 5.5 and 5.7 below for explicit expressions of $\left(M{ }_{F} C\right)_{S_{\lambda}}$ in some special cases.

Example 4.13 Let $W$ be a complex manifold of dimension $n^{\prime}$ and $M$ a complex submanifold of dimension $n$. Also let $V$ be an analytic subvariety of $W$ of dimension $k$. Recall that there exists a subanalytic triangulation of $W$ compatible with $M, V$ and $\operatorname{Sing}(V)$, the singular set of $V$. Thus, $V$ may be thought of as a chain, which is not $C^{\infty}$ but still has the associated 
current $T_{V}$ of integration. Moreover, it is a cycle, as the real codimension of $\operatorname{Sing}(V)$ in $V$ is greater than or equal to two. If $n+k=n^{\prime}$ and if $p$ is an isolated point of $M \cap V$, we have

$$
(M \cdot V)_{p} \geq \operatorname{mult}_{p}(V),
$$

the multiplicity of $V$ at $p$. The equality holds, by definition, if $M$ is general with respect to $V$, i.e., the intersection of the tangent space of $M$ at $p$ and the tangent cone of $V$ at $p$ consists only of $p$. Note that mult $p(V)$ coincides with the Lelong number of $T_{V}$ at $p$ (e.g., $[5$, Ch.3, 2]).

We finish this section by giving a formula for the residue at a non-isolated component. Thus, in the situation of Theorem 4.9, suppose that $S_{\lambda}$ is an oriented submanifold of $M$ of dimension $m-p$ with orientation compatible with that of $M$ in the sense described in Sect. 3.4 (b). Let $p_{\lambda}$ be a point in $S_{\lambda}$ and $B_{\lambda}$ a small open ball of dimension $p$ in $M$ transverse to $S_{\lambda}$ at $p_{\lambda}$. We orient $B_{\lambda}$ so that the orientation of $B_{\lambda}$ followed by that of $S_{\lambda}$ gives the orientation of $M$. Setting $F_{\lambda}=\left.F\right|_{B_{\lambda}}$, we have the commutative diagram

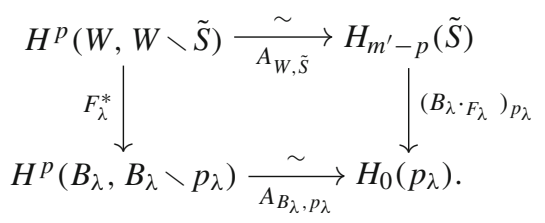

We have the residue $\operatorname{Res}\left(F_{\lambda}^{*} \Psi_{T}, p_{\lambda}\right)=\left(B_{\lambda} \cdot F_{\lambda} A\left(\Psi_{T}\right)\right)_{p_{\lambda}}$ in $H_{0}\left(p_{\lambda}\right) \simeq \mathbb{C}$ so that it is a number.

Theorem 4.14 In the situation of Theorem 4.9, suppose that $S_{\lambda}$ is an oriented submanifold of $M$ of dimension $m-p$ and let $p_{\lambda}$ and $F_{\lambda}$ be as above. Then we have:

$$
\operatorname{Res}\left(F^{*} \Psi_{T}, S_{\lambda}\right)=\operatorname{Res}\left(F_{\lambda}^{*} \Psi_{T}, p_{\lambda}\right) \cdot S_{\lambda} \quad \text { in } H_{m-p}\left(S_{\lambda}\right) \text {. }
$$

Proof We try to find $\operatorname{Res}\left(F^{*} \Psi_{T}, S_{\lambda}\right)$ by Proposition 4.11. As $U_{\lambda}$, we take a tubular neighborhood of $S_{\lambda}$ with a $C^{\infty}$ projection $\pi: U_{\lambda} \rightarrow S_{\lambda}$, which gives $U_{\lambda}$ the structure of a bundle of open balls of dimension $p$. Setting $U_{0}=U_{\lambda} \backslash S_{\lambda}$, we consider the covering $\mathcal{U}_{\lambda}=\left\{U_{0}, U_{\lambda}\right\}$ of $U_{\lambda}$. As $R_{\lambda}$, we take a bundle on $S_{\lambda}$ of closed balls of dimension $p$ in $U_{\lambda}$. Then $R_{0 \lambda}$ is a bundle on $S_{\lambda}$ of $(p-1)$-spheres. We denote the restrictions of $\pi$ to $R_{\lambda}$ and $R_{0 \lambda}$ by $\pi_{\lambda}$ and $\pi_{0 \lambda}$, respectively. For a closed $(m-p)$-form $\varphi$ on $U_{\lambda}$, we compute the integral

$$
I:=\int_{R_{\lambda}} F^{*} \psi_{1} \wedge \varphi+\int_{R_{0 \lambda}} F^{*} \psi_{01} \wedge \varphi .
$$

Since $\pi$ induces an isomorphism $\pi^{*}: H_{\mathrm{dR}}^{m-p}\left(S_{\lambda}\right) \stackrel{\sim}{\rightarrow} H_{\mathrm{dR}}^{m-p}\left(U_{\lambda}\right)$, there exist a closed $(m-p)$-form $\theta$ on $S_{\lambda}$ and an $(m-p-1)$-form $\tau$ on $U_{\lambda}$ such that

$$
\varphi=\pi^{*} \theta+\mathrm{d} \tau \text {. }
$$

Using the projection formula, the fact that $\mathrm{d} F^{*} \psi_{1}=0$ and the Stokes formula, we have

$$
\int_{R_{\lambda}} F^{*} \psi_{1} \wedge \varphi=\int_{S_{\lambda}}\left(\pi_{\lambda}\right)_{*} F^{*} \psi_{1} \cdot \theta+(-1)^{p+1} \int_{R_{0 \lambda}} F^{*} \psi_{1} \wedge \tau,
$$

where $\left(\pi_{\lambda}\right)_{*}$ denotes the integration along the fiber of $\pi_{\lambda}$. Note that $\left(\pi_{\lambda}\right)_{*} F^{*} \psi_{1}$ is a $C^{\infty}$ function on $S_{\lambda}$. Noting that $d F^{*} \psi_{01}=F^{*} \psi_{1}$ on $U_{0 \lambda}$ and $\partial R_{0 \lambda}=\emptyset$, we also compute to get

$$
\int_{R_{0 \lambda}} F^{*} \psi_{01} \wedge \varphi=\int_{S_{\lambda}}\left(\pi_{0 \lambda}\right)_{*} F^{*} \psi_{01} \cdot \theta+(-1)^{p} \int_{R_{0 \lambda}} F^{*} \psi_{1} \wedge \tau .
$$


Thus, we have

$$
I=\int_{S_{\lambda}}\left(\left(\pi_{\lambda}\right)_{*} F^{*} \psi_{1}+\left(\pi_{0 \lambda}\right)_{*} F^{*} \psi_{01}\right) \cdot \theta
$$

Now recall that we have the integration along the fiber on the $\breve{C} \mathrm{dR}$ cochains :

$$
\pi_{*}: A^{q}\left(\mathcal{U}_{\lambda}, U_{0}\right) \longrightarrow A^{q-p}\left(S_{\lambda}\right),
$$

which assigns to $\sigma=\left(0, \sigma_{\lambda}, \sigma_{0 \lambda}\right)$ the form $\left(\pi_{\lambda}\right)_{*} \sigma_{\lambda}+\left(\pi_{0 \lambda}\right)_{*} \sigma_{0 \lambda}$ on $S_{\lambda}$. Moreover, it is compatible with the differentials $D$ and $d$ (cf. [12, Ch.II, 5]). Since $\left(0,\left.F^{*} \psi_{1}\right|_{U_{\lambda}},\left.F^{*} \psi_{01}\right|_{U_{0 \lambda}}\right)$ is a C̆dR cocycle in $A^{p}\left(\mathcal{U}_{\lambda}, U_{0}\right)$, the function $\left(\pi_{\lambda}\right)_{*} F^{*} \psi_{1}+\left(\pi_{0 \lambda}\right)_{*} F^{*} \psi_{01}$ is $d$-closed so that it is a constant. By definition, the constant is exactly $\operatorname{Res}\left(F_{\lambda}^{*} \Psi_{T}, p_{\lambda}\right)$ above. Finally from

$$
\int_{S_{\lambda}} \theta=\int_{S_{\lambda}} \varphi
$$

we have the theorem.

Remark 4.15 1. In the above situation, $H_{m-p}\left(S_{\lambda}, \mathbb{Z}\right) \simeq \mathbb{Z}$ and is generated by the fundamental class $S_{\lambda}$. Thus if $\operatorname{Res}\left(F_{\lambda}^{*} \Psi_{T}, p_{\lambda}\right)$ is an integer, $\operatorname{Res}\left(F^{*} \Psi_{T}, S_{\lambda}\right)$ is an integral class.

2. The above theorem can also be proved topologically as [13, Theorem 4.1.1, see also Theorem 7.3.2]. In fact, using techniques and results in [13], we may compute residues in various settings.

\section{Coincidence point theorem}

\subsection{Coincidence homology classes and indices}

Let $M$ and $N$ be connected and oriented $C^{\infty}$ manifolds of dimensions $m$ and $n$, respectively, with $m \geq n$. We set $W=M \times N$ and orient $W$ so that the orientation of $M$ followed by that of $N$ gives the orientation of $W$. Let $f, g: M \rightarrow N$ be $C^{\infty}$ maps and denote by $\Gamma_{f}$ and $\Gamma_{g}$ the graphs of $f$ and $g$ in $W$. We consider the map

$$
\tilde{f}: M \longrightarrow \Gamma_{f} \subset W \quad \text { defined by } \tilde{f}(x)=(x, f(x)),
$$

which is a diffeomorphism onto $\Gamma_{f}$. We orient $\Gamma_{f}$ so that $\tilde{f}$ is orientation preserving. Similarly, we define $\tilde{g}$ for $g$. Recall the diagram (cf. Definition 4.7):

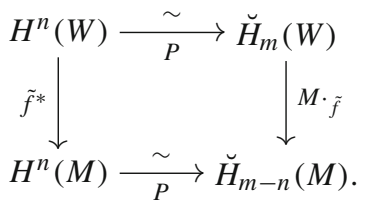

Definition 5.1 The global coincidence class $\Lambda(f, g)$ of the pair $(f, g)$ is defined by

$$
\Lambda(f, g)=M \cdot \tilde{f}\left[\Gamma_{g}\right] \quad \text { in } \breve{H}_{m-n}(M) .
$$

Note that $\tilde{f}$ induces an isomorphism $\tilde{f}_{*}: \breve{H}_{m-n}(M) \stackrel{\sim}{\rightarrow} \breve{H}_{m-n}\left(\Gamma_{f}\right)$ and $\Lambda(f, g)$ corresponds to $\Gamma_{f} \cdot\left[\Gamma_{g}\right]$ in $\breve{H}_{m-n}\left(\Gamma_{f}\right)$, which is sent to $\left[\Gamma_{f}\right] \cdot\left[\Gamma_{g}\right]$ in $\breve{H}_{m-n}(W)$ by the canonical homomorphism $\breve{H}_{m-n}\left(\Gamma_{f}\right) \rightarrow \breve{H}_{m-n}(W)$. 
We define the coincidence point set of the pair $(f, g)$ by

$$
\operatorname{Coin}(f, g)=\{p \in M \mid f(p)=g(p)\} .
$$

Note that Coin $(f, g)=\tilde{f}^{-1}\left(\Gamma_{g}\right)$. For shortness, we set $S=\operatorname{Coin}(f, g)$.

From now on, we assume that $M$ is compact so that $\Gamma_{g}$ and $S$ are compact. Recall the diagram (cf. Definition 4.7):

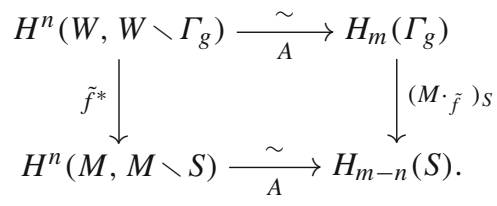

Definition 5.2 The local coincidence class $\Lambda(f, g ; S)$ of the pair $(f, g)$ at $S$ is defined to be the localized intersection class :

$$
\Lambda(f, g ; S)=\left(M \cdot \tilde{f} \Gamma_{g}\right)_{S} \quad \text { in } H_{m-n}(S) .
$$

Note that $\tilde{f}$ induces a homomorphism $\tilde{f}_{*}: H_{m-n}(S) \rightarrow H_{m-n}\left(\Gamma_{f}\right)$ and $\Lambda(f, g ; S)$ is sent to $\Gamma_{f} \cdot\left[\Gamma_{g}\right]$.

Remark 5.3 1. The classes $\Lambda(f, g)$ and $\Lambda(f, g ; S)$ are in fact in homology with $\mathbb{Z}$ coefficients.

2. We have

$$
\Lambda(g, f)=(-1)^{n} \Lambda(f, g), \quad \Lambda(g, f ; S)=(-1)^{n} \Lambda(f, g ; S) .
$$

Suppose $S=\operatorname{Coin}(f, g)$ has a finite number of connected components $\left(S_{\lambda}\right)_{\lambda}$. Then we have $H_{m-n}(S)=\bigoplus_{\lambda} H_{m-n}\left(S_{\lambda}\right)$, and accordingly, we have the local coincidence class $\Lambda\left(f, g ; S_{\lambda}\right)$ in $H_{m-n}\left(S_{\lambda}\right)$. From Theorem 4.9, we have a general coincidence point theorem:

Theorem 5.4 In the above situation

$$
\Lambda(f, g)=\sum_{\lambda}\left(i_{\lambda}\right)_{*} \Lambda\left(f, g ; S_{\lambda}\right) \quad \text { in } H_{m-n}(M) .
$$

In general, $\Lambda(f, g)$ and $\Lambda\left(f, g ; S_{\lambda}\right)$ are given as in Propositions 4.10 and 4.11. The theorem becomes more meaningful if we have explicit descriptions of them.

In the case $m=n, \Lambda\left(f, g ; S_{\lambda}\right)$ is in $H_{0}\left(S_{\lambda}\right)=\mathbb{C}$ so that it is a number (in fact an integer), which we call the coincidence index of $(f, g)$ at $S_{\lambda}$. If $S_{\lambda}$ consists of a point $p$, we have the following explicit formula. In fact it is already known; however, we give an alternative short proof using the Thom class in the Čech-de Rham cohomology. Let $U$ be a coordinate neighborhood around $p$ with coordinates $x=\left(x_{1}, \ldots, x_{m}\right)$ in $M$ and $V$ a coordinate neighborhood around $f(p)=g(p)$ with coordinates $y=\left(y_{1}, \ldots, y_{m}\right)$ in $N$. Also let $D$ be a closed ball around $p$ in $U$ such that $f(D) \subset V$ and $g(D) \subset V$. Thus, we may consider the map $g-f: D \rightarrow \mathbb{R}^{m}$ whose image is the origin 0 in $\mathbb{R}^{m}$ only at $p$. The boundary $\partial D$ is homeomorphic to the unit sphere $S^{m-1}$ and we have the map

$$
\gamma: \partial D \longrightarrow S^{m-1} \quad \text { defined by } \gamma(x)=\frac{g(x)-f(x)}{\|g(x)-f(x)\|} .
$$

We denote the degree of this map by $\operatorname{deg}(g-f, p)$. 
Proposition 5.5 In the above situation

$$
\Lambda(f, g ; p)=\operatorname{deg}(g-f, p) .
$$

Proof Let $\Psi_{\Gamma_{g}}$ be the Thom class of $\Gamma_{g}$ and $\left(0, \psi_{1}, \psi_{01}\right)$ its ČdR representative. We may take $D$ as $R_{\lambda}$ in Proposition 4.11. Since $R_{0 \lambda}=-\partial D$, we have

$$
\Lambda(f, g ; p)=\int_{D} \tilde{f}^{*} \psi_{1}-\int_{\partial D} \tilde{f}^{*} \psi_{01} .
$$

Recall that $\Psi_{\Gamma_{g}}$ may be naturally identified with the Thom class of the normal bundle $N_{\Gamma_{g}}$, which is trivial over $\tilde{g}(U) ; N_{\Gamma_{g}} \mid \tilde{g}(U) \simeq \mathbb{R}^{m} \times \tilde{g}(U)$ (cf. Sect. 3.4b]. Let $\rho_{g}: N_{\Gamma_{g}} \mid \tilde{g}(U) \rightarrow \mathbb{R}^{m}$ denote the projection onto the fiber direction. Also let $\psi_{m}$ be an angular form on $\mathbb{R}^{m} \backslash\{0\}$. Then on $\tilde{g}(U)$ the Thom class of $N_{\Gamma_{g}}$ is represented by the cocycle (cf. Proposition 3.3)

$$
\left(0,0,-\rho_{g}^{*} \psi_{m}\right) \text {. }
$$

Let $\pi_{1}$ and $\pi_{2}$ denote the projections of $W=M \times N$ onto the first and second factors, respectively. We set $x=\pi_{1}^{*} x$ and $y=\pi_{2}^{*} y$ on $U \times V$. By our orientation convention, in a neighborhood of $\tilde{g}(p)$ in $N_{\Gamma_{g}}$, we may take $g(x)-y$ as fiber coordinates and $x$ as base coordinates of the bundle $N_{\Gamma_{g}}$ so that we may write $\rho_{g}(x, y)=g(x)-y$. Then (5.1) becomes

$$
\Lambda(f, g ; p)=\int_{\partial D}\left(\rho_{g} \circ \tilde{f}\right)^{*} \psi_{m}=\int_{\partial D}(g-f)^{*} \psi_{m},
$$

which is nothing but $\operatorname{deg}(g-f, p)$.

In the above situation, let $J_{f}(p)$ and $J_{g}(p)$ denote the Jacobian matrices of $f$ and $g$ at $p$. A coincidence point $p$ of the pair $(f, g)$ is said to be non-degenerate if

$$
\operatorname{det}\left(J_{g}(p)-J_{f}(p)\right) \neq 0 .
$$

Corollary 5.6 If $p$ is a non-degenerate coincidence point,

$$
\Lambda(f, g ; p)=\operatorname{sgn} \operatorname{det}\left(J_{g}(p)-J_{f}(p)\right) .
$$

Now consider the case $m>n$. Suppose $S_{\lambda}$ is an oriented submanifold of $M$ of dimension $m-n$. Let $p_{\lambda}$ be a point in $S_{\lambda}$ and $B_{\lambda}$ a small open ball of dimension $n$ in $M$ transverse to $S_{\lambda}$ at $p_{\lambda}$. Setting $f_{\lambda}=\left.f\right|_{B_{\lambda}}$ and $g_{\lambda}=\left.g\right|_{B_{\lambda}}$, we have $\operatorname{deg}\left(g_{\lambda}-f_{\lambda}, p_{\lambda}\right)$. From Theorem 4.14, we have

Proposition 5.7 Let $S_{\lambda}$ be a connected component of Coin $(f, g)$. If $S_{\lambda}$ is an oriented submanifold of $M$ of dimension $m-n$,

$$
\Lambda\left(f, g ; S_{\lambda}\right)=\operatorname{deg}\left(g_{\lambda}-f_{\lambda}, p_{\lambda}\right) \cdot S_{\lambda} \quad \text { in } H_{m-n}\left(S_{\lambda}\right) .
$$

\subsection{Lefschetz coincidence point formula}

Let $M$ and $N$ be compact, connected and oriented $C^{\infty}$ manifolds of the same dimension $m$ and let $f, g: M \rightarrow N$ be $C^{\infty}$ maps. In this situation, $\breve{H}_{0}(M)=H_{0}(M)=\mathbb{C}$ and $\Lambda(f, g)$ is a number (in fact an integer), which has an explicit description. Let

$$
H^{p}(f): H^{p}(N) \longrightarrow H^{p}(M)
$$

be the homomorphism induced by $f$ on the $p$-th cohomology group and similarly for $H^{p}(g)$. We set $q=m-p$. The Poincaré duality allows us to define the composition

$$
H^{q}(M) \simeq H^{p}(M)^{*} \stackrel{H^{p}(g)^{*}}{\longrightarrow} H^{p}(N)^{*} \simeq H^{q}(N) \stackrel{H^{q}(f)}{\longrightarrow} H^{q}(M) .
$$


We define the Lefschetz, coincidence number $L(f, g)$ of the pair $(f, g)$ as

$$
L(f, g):=\sum_{q=0}^{m}(-1)^{q} \cdot \operatorname{tr}\left(H^{q}(f) \circ H^{m-q}(g)^{*}\right) .
$$

Although the following is already known, we include a proof for the sake of completeness. It is a modification of the presentation as given in [5] for the fixed point case, i.e., the case $M=N$ and $g=1_{M}$, the identity map of $M$.

Proposition 5.8 In the above situation, we have

$$
\Lambda(f, g)=L(f, g) .
$$

Proof Let $\left\{\mu_{i}^{p}\right\}_{i}$ be a set of closed forms representing a basis of $H_{\mathrm{dR}}^{p}(M)$. We set $q=m-p$ and let $\left\{\check{\mu}_{j}^{q}\right\}_{j}$ be a set of forms representing a basis of $H_{\mathrm{dR}}^{q}(M)$ dual to $\left\{\left[\mu_{i}^{p}\right]\right\}_{i}$ :

$$
\int_{M} \mu_{i}^{p} \wedge \check{\mu}_{j}^{q}=\delta_{i j}
$$

We also take a set of forms $\left\{v_{k}^{p}\right\}_{k}$ representing a basis of $H_{\mathrm{dR}}^{p}(N)$ and a set of forms $\left\{\check{v}_{\ell}^{q}\right\}_{\ell}$ representing a basis of $H_{\mathrm{dR}}^{q}(N)$ dual to $\left\{\left[v_{k}^{p}\right]\right\}_{k}$. By the Künneth formula, a basis of $H_{\mathrm{dR}}^{m}(W)$, $W=M \times N$, is represented by

$$
\left\{\xi_{i, \ell}^{p, q}=\pi_{1}^{*} \mu_{i}^{p} \wedge \pi_{2}^{*} \check{\nu}_{\ell}^{q}\right\}_{p+q=m}
$$

where $\pi_{1}$ and $\pi_{2}$ are projections onto the first and second factors.

Note that in general, for a $p$-form $\omega$ on $M$ and a $q$-form $\theta$ on $N$, we have

$$
\int_{\Gamma_{f}} \pi_{1}^{*} \omega \wedge \pi_{2}^{*} \theta=\int_{M} \omega \wedge f^{*} \theta
$$

and similarly for the integration on $\Gamma_{g}$.

Let $G^{p}=\left(g_{k i}^{p}\right)$ be the matrix representing $H^{p}(g)$ in the bases $\left\{\left[v_{k}^{p}\right]\right\}_{k}$ and $\left\{\left[\mu_{i}^{p}\right]\right\}_{i}$ :

$$
H^{p}(g)\left[v_{k}^{p}\right]=\sum_{i} g_{i k}^{p}\left[\mu_{i}^{p}\right] .
$$

Thus, the dual map $H^{p}(g)^{*}$ is represented by the transposed ${ }^{t} G^{p}$ in the bases $\left\{\left[\check{\mu}_{j}^{q}\right]\right\}_{j}$ and $\left\{\left[\check{v}_{\ell}^{q}\right]\right\}_{\ell}$. Also let $\check{F}^{q}=\left(\check{f}_{\ell j}^{q}\right)$ be the matrix representing $H^{q}(f)$ in the bases $\left\{\left[\check{v}_{\ell}^{q}\right]\right\}_{\ell}$ and $\left\{\left[\check{\mu}_{j}^{q}\right\}_{j}\right.$ :

$$
H^{q}(f)\left[\check{v}_{\ell}^{q}\right]=\sum_{j} \check{f}_{j \ell}^{q}\left[\check{\mu}_{j}^{q}\right] .
$$

Let $\eta_{g}$ be an $m$-form representing the Poincaré dual of $\left[\Gamma_{g}\right]$ in $W$. Using (3.3) and (5.2) for $\Gamma_{g}$, we compute to get

$$
\left[\eta_{g}\right]=\sum_{q, i, \ell}(-1)^{q} g_{i \ell}^{p}\left[\xi_{i, \ell}^{p, q}\right]
$$

Thus, we have

$$
\Lambda(f, g)=\int_{M} \tilde{f}^{*} \eta_{g}=\sum_{q, i, \ell}(-1)^{q} g_{i \ell}^{p} \int_{M} \mu_{i}^{p} \wedge f^{*} \check{v}_{\ell}^{q}=\sum_{q, i, \ell}(-1)^{q} g_{i \ell}^{p} \check{f}_{i \ell}^{q} .
$$

Since $\check{f}_{i \ell}^{q}$ is the $\ell i$ entry of $\check{F}^{q}$ and $g_{i \ell}^{p}$ the $i \ell$ entry of ${ }^{t} G^{p}$, we have the proposition. 
From Theorem 5.4 and Propositions 5.5 and 5.8, we have

Theorem 5.9 Let $M$ and $N$ be compact oriented $C^{\infty}$ manifolds of same dimension and let $f, g: M \rightarrow N$ be $C^{\infty}$ maps. Suppose $\operatorname{Coin}(f, g)$ has a finite number of connected components $\left(S_{\lambda}\right)_{\lambda}$. Then

$$
L(f, g)=\sum_{\lambda} \Lambda\left(f, g ; S_{\lambda}\right)
$$

In the case the set of coincidence points consists only of isolated points, we have

Corollary 5.10 (Lefschetz coincidence point formula) Let $M$ and $N$ be compact oriented $C^{\infty}$ manifolds of the same dimension and let $f, g: M \rightarrow N$ be $C^{\infty}$ maps. Suppose Coin $(f, g)$ consists of a finite number of isolated points. Then

$$
L(f, g)=\sum_{p \in \operatorname{Coin}(f, g)} \operatorname{deg}(g-f, p) .
$$

Moreover if all coincidence points are isolated and non-degenerate, then

$$
L(f, g)=\sum_{p \in \operatorname{Coin}(f, g)} \operatorname{sgn} \operatorname{det}\left(J_{g}(p)-J_{f}(p)\right) .
$$

Remark 5.11 1. The above theory applied to the case $N=M$ and $g=1_{M}$, the identity map of $M$, gives a general fixed point theorem for $f$ and the Lefschetz fixed point formula, which is effective also in the study of periodic points.

2. Let $f, g: M \rightarrow N$ be $C^{\infty}$ maps. If $g$ is a diffeomorphism, the coincidence theory for the pair $(f, g)$ is equivalent to the fixed point theory for the map $g^{-1} \circ f$ of $M$.

3. As a textbook dealing with the Lefschetz coincidence theory, we list [14].

4. In [10], for a pair of maps from a topological space to a manifold, the Lefschetz homomorphism is defined and it is proved that if it is non-trivial, then there is a coincidence point.

\section{References}

1. Bott, R.: Lectures on Characteristic Classes and Foliations, Lectures on Algebraic and Differential Topology, Lecture Notes in Math, vol. 279, pp. 1-94. Springer, Berlin (1972)

2. Bott, R., Tu, L.: Differential Forms in Algebraic Topology, Graduate Texts in Math, vol. 82. Springer, Berlin (1982)

3. Brasselet, J.-P.: Définition combinatoire des homomorphismes de Poincaré, Alexander et Thom pour une pseudo-variété. Astérisque Soc. Math. 82, 71-91 (1981)

4. Fulton, W.: Intersection Theory. Springer, Berlin (1984)

5. Griffiths, P., Harris, J.: Principles of Algebraic Geometry. Wiley, Hoboken (1978)

6. Lefschetz, S.: Intersections and transformations of complexes and manifolds. Trans. Am. Math. Soc. 28, $1-49$ (1926)

7. Lefschetz, S.: Manifolds with a boundary and their transformations. Trans. Am. Math. Soc. 29, 429-462 (1927)

8. Lehmann, D.: Systèmes d'alvéoles et intégration sur le complexe de Čech-de Rham, Publications de l'IRMA, vol. 23, N ${ }^{o}$ VI. Université de Lille I, Villeneuve-d'Ascq (1991)

9. Milnor, J., Stasheff, J.: Characteristic Classes, Annals of Math Studies, vol. 76. Princeton University Press, Princeton (1974)

10. Saveliev, P.: Lefschetz coincidence theory for maps between spaces of different dimensions. Topol. Appl. 116, 137-152 (2001)

11. Shiota, M.: Geometry of Subanalytic and Semialgebraic Sets, Progress in Math. Birkhäuser, Boston (1997) 
12. Suwa, T.: Indices of Vector Fields and Residues of Singular Holomorphic Foliations. Hermann, Paris (1998)

13. Suwa, T.: Residue Theoretical Approach to Intersection Theory. In: Workshop on Real and Complex Singularities. Contemp. Math., Am. Math. Soc. 459, 207-261 (2008)

14. Vick, J.W.: Homology Theory: An Introduction to Algebraic Topology, Graduate Texts in Math. Springer, Berlin (1994) 